\title{
Dynamically generated gap from holography in the charged black brane with hyperscaling violation
}

\author{
Xiao-Mei Kuang, ${ }^{a, b}$ Eleftherios Papantonopoulos, ${ }^{a, c}$ Bin Wang ${ }^{d}$ and Jian-Pin Wu ${ }^{e, f}$ \\ ${ }^{a}$ Department of Physics, National Technical University of Athens, \\ Athens, GR-15780 Greece \\ ${ }^{b}$ Instituto de Física, Pontificia Universidad Católica de Valparaíso, \\ Casilla 4059, Valparaiso, Chile \\ ${ }^{c}$ CERN - Theory Division, \\ Geneva 23, CH-1211 Switzerland \\ ${ }^{d}$ IFSA Collaborative Innovation Center, Department of Physics and Astronomy, \\ Shanghai Jiao Tong University, Shanghai, 200240 China \\ ${ }^{e}$ Department of Physics, School of Mathematics and Physics, Bohai University, \\ Jinzhou, 121013 China \\ ${ }^{f}$ State Key Laboratory of Theoretical Physics, Institute of Theoretical Physics, \\ Chinese Academy of Sciences, Beijing, 100190 China \\ E-mail: xmeikuang@gmail.com, lpapa@central.ntua.gr, \\ wang_b@sjtu.edu.cn, jianpinwu@gmail.com
}

ABSTRACT: We holographically investigate the effects of a dipole coupling between a fermion field and a U(1) gauge field on the dual fermionic sector in the charged gravity bulk with hyperscaling violation. We analytically study the features of the ultraviolet and infrared Green's functions of the dual fermionic system and we show that as the dipole coupling and the hyperscaling violation exponent are varied, the fluid possess Fermi, marginal Fermi, non-Fermi liquid phases and also an additional Mott insulating phase. We find that the increase of the hyperscaling violation exponent which effectively reduces the dimensionality of the system makes it harder for the Mott gap to be formed. We also show that the observed duality between zeros and poles in the presence of a dipole moment coupling still persists in theories with hyperscaling violation.

KEYworDs: AdS-CFT Correspondence, Holography and condensed matter physics $(\mathrm{AdS} / \mathrm{CMT})$

ARXIV EPRINT: 1411.5627 


\section{Contents}

1 Introduction 1

2 The charged black branes with hyperscaling violation from EinsteinMaxwell-Dilaton theory 3

3 The holographic fermionic model $\quad 6$

3.1 The Dirac equation 6

$\begin{array}{ll}3.2 \text { Green's function } & 7\end{array}$

$\begin{array}{lll}3.2 .1 & \text { UV limit } & 7\end{array}$

3.2 .2 IR limit 8

4 The effect of dipole coupling on the spectral function in the Fermionic $\begin{array}{ll}\text { system } & 10\end{array}$

4.1 The emergence of the Mott gap 10

4.2 The formation of the Fermi surface and the type of low energy excitations 12

5 The zeros-poles duality of the holographic system 14

6 Conclusions and discussion $\quad 15$

\section{Introduction}

The gauge/gravity duality is a powerful principle which describes many physical systems holographically. This holographic description relates strongly-coupled quantum field theories with their classical gravitational counterparts which live in one higher dimension [1-3]. A remarkable implementation of this holographic principle was realized in investigating strongly coupled systems in condensed matter physics, the so called AdS/CMT correspondence, which made possible the better understanding of numerous exotic but very important features of electronic materials, including the high temperature superconductors and the heavy fermion systems.

To describe these systems holographycally, fermion fields coupled to U(1) gauge field have to be introduced in the gravity sector. The fermions were treated as a probe and their backreaction on the background geometry were ignored [4-7]. The properties of the spectral function showed that a Fermi surface usually emerges, the low energy excitations of it can exhibit Fermi liquid, marginal Fermi liquid, or non-Fermi liquid behaviors. To describe better various phases of a metallic state at low temperatures, a dipole coupling to massless charged fermions was introduced $[8,9]$. The presence of the dipole moment introduced a scale in the system and this enabled in the dual field theory to model a Mott insulating phase, generate dynamically a gap and spectral weight transfer. This proposal 
has triggered further interest in the study of the dipole coupling effect on the holographic fermionic systems [10-19]. Then it was found in [20] that there exists a duality between zeroes and poles in holographic systems with massless fermions and a dipole coupling, which was also observed in [21].

In many condensed matter materials it was observed that at criticality their scaling properties go beyond the standard Lorentz scaling. Then there is a need to describe holographically these systems with anisotropic (Lifshitz) scaling characterized by the dynamic critical exponent $z>1$ [22], or even with hyperscaling violation characterized by a non-zero hyperscaling violation exponent $\theta[23-25]$.

To formulate the duality principle to describe holographically of such systems, it was proposed [26] that their gravity duals should have a metric of the form

$$
d s^{2}=r^{\frac{-2 \theta}{d}}\left(-r^{2 z} d t^{2}+\frac{d r^{2}}{r^{2}}+r^{2} d x_{i}^{2}\right) .
$$

Under the transformation

$$
t \rightarrow \lambda^{z} t, \quad x_{i} \rightarrow \lambda x_{i}, \quad r \rightarrow \lambda^{-1} r,
$$

with $z \neq 1$ indicating an anisotropy between time and space. The metric (1.1) transforms as $d s \rightarrow \lambda^{\theta / d} d s$ which breaks the scale-invariance. A non-zero $\theta$, indicates a hyperscaling violation in the dual field theory. This metric is characterized by dynamical critical exponent $z$ and hyperscaling violation exponent $\theta[27]$ and when $\theta=0$ and $z \neq 1$, it is reduced to the Lifshitz metric [22, 28-30], while it describes the pure AdS metric when $\theta=0$ and $z=1$.

Thermodynamically in these theories the entropy scales as $T^{(d-\theta) / z}$, while in theories with gravity duals having the standard AdS metric, the entropy scales as $T^{d}$. Note that the hyperscaling violation leads to an effective dimension $d_{\theta}=d-\theta$. It was addressed in [31] that with the critical value $d-\theta=1$, the entanglement entropy shows up a logarithmic violation $[32,33]$, and leads to an infrared metric which holographically represents a compressible state with hidden Fermi surfaces [24].

The introduction of Lifschitz scaling and hyperscaling violation exponents in the metric of the gravity sector has produced interesting results. It was showed that these critical exponents play important role in the retarded Green's function in holographic systems with finite charged density [34-36]. It was found in [37] that for a specific value of the critical exponent $z$, the Luttinger's theorem is violated. In a dual charged bulk theory with hyperscaling violation, introducing a charge fermion as a probe to the extremal gravity background, it was showed in [36] that the increase of the Lifshitz factor $z$ and the hyperscaling factor $\theta$ broadened and smoothed out the sharp spectral function's peak, which indicates that the system does not have a Fermi surface.

In this work we will consider a dipole coupling in a charged gravity bulk with hyperscaling violation and explore the spectral function of the holographic dual fermion model. The dual model with minimal coupling between fermionic field and gauge field was discussed in our previous work [38], in which we showed that as the hyperscaling violation exponent is varied, the fermionic system possesses Fermi, non-Fermi, marginal-Fermi and 
log-oscillating liquid phases but failed to generate dynamically a gap. In the case of Lifshitz geometry, the generation of Mott gap due to the dipole coupling has been observed in $[39-41]$.

Our aim here is to study in details the behaviour of infrared (IR) and ultra violet (UV) Green's functions in an attempt to understand how the hyperscaling exponent modifies the dipole effect on the formation of Fermi surface, the liquid types of the low energy excitations and the emergence of the Mott insulating phase in these theories. Finally, we will show that there is a duality of the zeros and the poles, first observed in [20], in theories with hyperscaling violation.

The work is organized as follows. In section 2 we review the charged black hole background with hyperscaling factor and analyze the geometry in the near horizon limit at zero temperature. We set up the fermionic model and analytically study the Green's function in the bulk theory in section 3. In section 4 we numerically investigate the properties of the UV Green's function and we discuss the effect of the hyperscaling exponent on the emergerence of the gap, the formation of Fermi momentum and the excitations due to the dipole coupling. Finally in section 6 we present our conclusions.

\section{The charged black branes with hyperscaling violation from Einstein- Maxwell-Dilaton theory}

In order to study the dipole coupling effects on the charged black brane geometry with hyperscaling violation, we will consider the $(3+1)$-dimensional Einstein-Maxwell-Dilaton action [30]

$$
S_{g}=-\frac{1}{16 \pi G} \int \mathrm{d}^{4} x \sqrt{-g}\left[R-\frac{1}{2}(\partial \phi)^{2}+V(\phi)-\frac{1}{4}\left(e^{\lambda_{1} \phi} F^{\mu \nu} F_{\mu \nu}+e^{\lambda_{2} \phi} \mathcal{F}^{\mu \nu} \mathcal{F}_{\mu \nu}\right)\right]
$$

which contains two U(1) gauge fields coupled to a neutral scalar field $\phi$. The U(1) field $A$ with field strength $F_{\mu \nu}$ is necessary to generate a charged black brane solution, while the other gauge field $\mathcal{A}$ with field strength $\mathcal{F}_{\mu \nu}$ is required to generalize the geometry from AdS to the one with the hyperscaling violation. Following [36] and our discussion in [38], if one considers a potential of the form

$$
V(\phi)=V_{0} e^{\gamma \phi}
$$

which is necessary to obtain a general Lifshitz form of the metric with hyperscaling violation, one can find the charged black brane solution with hyperscaling violation [36]

$$
\begin{aligned}
d s_{4}^{2} & =r^{-\theta}\left(-r^{2 z} f(r) d t^{2}+\frac{d r^{2}}{r^{2} f(r)}+r^{2}\left(d x^{2}+d y^{2}\right)\right), \\
f & =1-\left(\frac{r_{\mathrm{h}}}{r}\right)^{2+z-\theta}+\frac{Q^{2}}{r^{2(z-\theta+1)}}\left[1-\left(\frac{r_{\mathrm{h}}}{r}\right)^{\theta-z}\right], \\
\mathcal{A}_{t} & =-\mu r_{\mathrm{h}}^{2+z-\theta}\left[1-\left(\frac{r}{r_{\mathrm{h}}}\right)^{2+z-\theta}\right],
\end{aligned}
$$




$$
\begin{aligned}
& A_{t}=\mu r_{\mathrm{h}}^{\theta-z}\left[1-\left(\frac{r_{\mathrm{h}}}{r}\right)^{z-\theta}\right] \\
& e^{\phi}=e^{\phi_{0}} r^{\sqrt{2(2-\theta)(z-1-\theta / 2)}}
\end{aligned}
$$

where we have defined

$$
\begin{aligned}
& \mu=\frac{\sqrt{2(z-1)(2+z-\theta)}}{2+z-\theta} e^{\frac{2-\theta / 2}{\sqrt{2(2-\theta)(z-1-\theta / 2)}} \phi_{0}}, \\
& \mu=Q \sqrt{\frac{2(2-\theta)}{z-\theta}} e^{-\sqrt{\frac{z-1+\theta / 2}{2(2-\theta)}} \phi_{0}} .
\end{aligned}
$$

Here, $r_{\mathrm{h}}$ is the radius of horizon satisfying $f\left(r_{\mathrm{h}}\right)=0$ and $Q=\frac{1}{16 \pi G} \int e^{\lambda_{1} \phi} F_{r t}$ is the total charge of the black brane. All the parameters in the action, dependent on the Lifshitz scaling exponent $z$ and hyperscaling violation exponents $\theta$, and they can be determined as

$$
\begin{aligned}
\lambda_{1} & =\sqrt{\frac{2(z-1-\theta / 2)}{2-\theta}}, \\
\lambda_{2} & =-\frac{2(2-\theta / 2)}{\sqrt{2(2-\theta)(z-\theta / 2-1)}}, \\
\gamma & =\frac{\theta}{\sqrt{2(2-\theta)(z-1-\theta / 2)}}, \\
V_{0} & =e^{\frac{-\theta \phi_{0}}{\sqrt{2(2-\theta)(z-1-\theta / 2)}}(z-\theta+1)(z-\theta+2) .}
\end{aligned}
$$

The Hawking temperature of the black hole is

$$
T=\frac{(2+z-\theta) r_{\mathrm{h}}^{z}}{4 \pi}\left[1-\frac{(z-\theta) Q^{2}}{2+z-\theta} r_{\mathrm{h}}^{2(\theta-z-1)}\right] .
$$

Note that we have $z \geq 1, \theta \geq 0$ and the above solutions are not valid for $\theta=2$. Before proceeding, we would like to remark more on the parameters $z$ and $\theta$. First, the background equations (2.3)-(2.7) are valid only for $z \geq 1$ and $\theta \geq 0$. The case of $z=1$ and $\theta=0$ corresponds to the AdS geometry. Second, the condition $z-\theta \geq 0$ is required to make chemical potential well-defined in the dual field theory. Third, it is easy to see that $\theta<2$ from equation (2.9). Combining the requirement of the null energy condition $\left(-\frac{\theta}{2}+1\right)\left(-\frac{\theta}{2}+z-1\right) \geq 0$ [36], one can have $\theta \leq 2(z-1)$. Thus, in this charged background, the range of the parameters is

$$
\begin{cases}0 \leq \theta \leq 2(z-1) & \text { for } \quad 1 \leq z<2 \\ 0 \leq \theta<2 & \text { for } \quad z \geq 2\end{cases}
$$

For convenience we introduce the following rescaling

$$
\begin{aligned}
& r \rightarrow r_{\mathrm{h}} r, \quad t \rightarrow \frac{t}{r_{\mathrm{h}}^{z}}, \quad(x, y) \rightarrow \frac{1}{r_{\mathrm{h}}}(x, y), \quad T \rightarrow \frac{T}{r_{\mathrm{h}}^{z}}, \\
& Q \rightarrow r_{\mathrm{h}}^{(z-\theta+1)} Q, \quad A_{t} \rightarrow r_{\mathrm{h}} A_{t}, \quad \mathcal{A}_{t} \rightarrow r_{\mathrm{h}}^{\theta-z-2} \mathcal{A}_{t},
\end{aligned}
$$


and we set $r_{\mathrm{h}}$ to be unity. Besides, we will set $\phi_{0}=0$ in the following discussion because it is an integration constant. Then we can rewrite the metric factor $f(r)$ and the gauge fields $\mathcal{A}_{t}$ and $A_{t}$ as follows

$$
\begin{aligned}
f & =1-\frac{1+Q^{2}}{r^{z+2-\theta}}+\frac{Q^{2}}{r^{2(z-\theta+1)}}, \\
\mathcal{A}_{t} & =-\mu\left[1-r^{2+z-\theta}\right], \\
A_{t} & =\mu\left[1-\left(\frac{1}{r}\right)^{z-\theta}\right],
\end{aligned}
$$

and the dimensionless temperature in the form

$$
T=\frac{(2+z-\theta)}{4 \pi}\left[1-\frac{(z-\theta) Q^{2}}{2+z-\theta}\right]
$$

The zero-temperature limit can reached when $Q=\sqrt{\frac{2+z-\theta}{z-\theta}}$ and $\mu=\frac{\sqrt{2(2-\theta)(2+z-\theta)}}{z-\theta}$. Thus at zero temperature and in the $r \rightarrow r_{\mathrm{h}}=1$ limit, we can reduce

$$
\left.f(r)\right|_{T=0, r \rightarrow 1} \simeq(z-\theta+1)(z-\theta+2)(r-1)^{2} \equiv \frac{1}{L_{2}^{2}}(r-1)^{2} .
$$

Therefore, at the zero temperature, we obtain the near horizon geometry $A d S_{2} \times \mathbb{R}^{2}$ with the curvature radius $L_{2} \equiv 1 / \sqrt{(z-\theta+1)(z-\theta+2)}$ of $A d S_{2}$ which depends explicitly on the Lifshitz scaling exponent $z$ and hyperscaling violation exponent $\theta$. So, near the horizon, under the transformation $r-1=\epsilon \frac{L_{2}^{2}}{\varsigma}$ and $t=\epsilon^{-1} \tau$ the metric and the gauge fields are derived in the limit $\epsilon \rightarrow 0$ with finite $\varsigma$ and $\tau$,

$$
\begin{array}{ll}
d s^{2}=\frac{L_{2}^{2}}{\varsigma^{2}}\left(-d \tau^{2}+d \varsigma^{2}\right)+d x^{2}+d y^{2}, & \\
\mathcal{A}_{\tau}=\frac{\notin}{\varsigma}, & A_{\tau}=\frac{e}{\varsigma},
\end{array}
$$

where $\phi=\mu(2+z-\theta) L_{2}^{2}$ and $e=\mu(z-\theta) L_{2}^{2}$.

Before proceeding to the study of holographic fermionic, we would like to give some comments on the geometry. We rewrite the Einstein-Maxwell-Dilaton action (2.1) as

$$
S_{g}=-\frac{1}{16 \pi G} \int \mathrm{d}^{4} x \sqrt{-g}\left[R-\frac{1}{4} Z(\phi) F^{\mu \nu} F_{\mu \nu}-\frac{1}{2}(\partial \phi)^{2}+V_{\text {eff }}(\phi)\right],
$$

where

$$
Z(\phi)=e^{\lambda_{1} \phi} \quad \text { and } \quad V_{\text {eff }}(\phi)=V(\phi)-\frac{1}{4} e^{\lambda_{2} \phi} \mathcal{F}^{\mu \nu} \mathcal{F}_{\mu \nu}=V_{0} e^{\gamma \phi}+V_{\mathcal{A}} e^{\gamma_{\mathcal{A}} \phi}
$$

with

$$
V_{\mathcal{A}}=\frac{1}{2}(z-1)(z+2-\theta) \quad \text { and } \quad \gamma_{\mathcal{A}}=\frac{\theta}{\sqrt{2(2-\theta)(z-1-\theta / 2)}} .
$$


It is easy to check that $\theta=0$ and $z=1$, leading to $Z(\phi)=1$ and $V_{\text {eff }}(\phi)=6$, give us the RN-AdS black hole solution. When $V_{\text {eff }}(\phi)=V_{0}+V_{\mathcal{A}} e^{\gamma_{\mathcal{A}} \phi}$, it was addressed in [30] that the action admits a Lifshitz black hole solution with $\theta=0$. Therefore, the effective potential $V_{\text {eff }}(\phi)$ controls the UV geometry, which can be changed from AdS to Lifshitz, even to the geometry with hyperscaling violation accompanying the parameters $\lambda_{1}$ and $\gamma_{\mathcal{A}}$.

The effective potential (2.21) is very different from that shown in $[23,26]$, in which the authors constructed an effective holographic theory with a scalar field $\phi$ and only one gauge field $A_{\mu}$. In their work, the parameters $\lambda_{1}$ in the gauge coupling function $Z(\phi)=e^{\lambda_{1} \phi}$ and $\gamma$ in the potential $V(\phi)=V_{0} e^{\gamma \phi}$ control the IR behavior. While for the UV limit, the potential $V(\phi)$ is required to vanish so that it usually leads to asymptotically AdS. When the gauge field in $[23,26]$ plays the role of $\mathcal{F}_{\mu \nu}$ but not that of $F_{\mu \nu}$ in the action $(2.1)$, one can obtain UV geometry with asymptotically Lifshitz-AdS but uncharged as discussed in [28].

\section{The holographic fermionic model}

\subsection{The Dirac equation}

To probe the geometry with hyperscaling violation, we consider the following Dirac action including a dipole moment coupling between the fermion and the gauge field ${ }^{1}$

$$
S_{D}=i \int d^{d+1} x \sqrt{-g} \bar{\zeta}\left(\Gamma^{a} \mathcal{D}_{a}-m-i p \not f\right) \zeta
$$

where $\mathcal{D}_{a}=\partial_{a}+\frac{1}{4}\left(\omega_{\mu \nu}\right)_{a} \Gamma^{\mu \nu}-i q A_{a}$ and $\not{F}=\frac{1}{2} \Gamma^{\mu \nu}\left(e_{\mu}\right)^{a}\left(e_{\nu}\right)^{b} F_{a b}$ with $\Gamma^{\mu \nu}=\frac{1}{2}\left[\Gamma^{\mu}, \Gamma^{\nu}\right]$ and the spin connection $\left(\omega_{\mu \nu}\right)_{a}=\left(e_{\mu}\right)^{b} \nabla_{a}\left(e_{\nu}\right)_{b}$. Here, it is worthwhile to point out that as a "bottom-up" approach, one can also add an additional dipole coupling term between the Dirac field and the other gauge field $\mathcal{A}$, i.e., $\tilde{p} \bar{\zeta} \mathcal{F} \zeta$ with $\mathcal{F}=\frac{1}{2} \Gamma^{\mu \nu}\left(e_{\mu}\right)^{a}\left(e_{\nu}\right)^{b} \mathcal{F}_{a b}$, into the Dirac action (3.1) and then explore its effects on the spectral function as well as the enhance/competition between $p$ and $\tilde{p}$. However, $\tilde{p} \bar{\zeta} \mathcal{F} \zeta$ will be divergent in the UV boundary. Therefore, a new boundary counterterm is usually needed to obtain a finite on-shell action. Ref. [49] can shed a light on how to understand the divergences from the point of view of the field theory and the boundary counterterms. We shall further explore this subject in the future. In this work, we will only investigate the case with $\tilde{p}=0$.

Now, we shall derive the Dirac equation. From the above action, with the redefinition $\zeta=\left(-g g^{r r}\right)^{-\frac{1}{4}} \mathcal{F}$ and a Fourier transformation $\mathcal{F}=F e^{-i \omega t+i k_{i} x^{i}}$, we can write the Dirac equation in the Fourier space

$$
\left(\sqrt{g^{r r}} \Gamma^{r} \partial_{r}-m\right) F-i\left(\omega+q A_{t}\right) \sqrt{g^{t t}} \Gamma^{t} F+i\left(k \sqrt{g^{x x}}-p \sqrt{g^{t t} g^{r r}} \partial_{r} A_{t}\right) \Gamma^{x} F=0 .
$$

\footnotetext{
${ }^{1}$ The Dirac action (3.1) depends on the effective chemical potential $\mu_{\mathrm{eff}} \equiv \mu q$ and the product of the dipole coupling $p$ and $\mu$. That is to say, the Dirac action depends on the combination of $g_{F} q$ with $g_{F}=\frac{2}{\sqrt{Z(\phi)}}$. For the case of $z=1$ and $\theta=0, g_{F} q=2$ because of $Z(\phi)=1$. It is different from refs. $[5,8,9]$, in which the authors set $g_{F}=1$. Therefore, we remind readers to note that the charge $q$ and the bulk dipole coupling $p$ for $z=1$ and $\theta=0$ here will correspond to $q / 2$ and $p / 2$ in refs. [5, 8, 9].
} 
Due to the rotational symmetry in $x-y$ plane, we have set $k_{x}=k$ and $k_{y}=0$. With the choice of the usual gamma matrices

$$
\Gamma^{r}=\left(\begin{array}{cc}
-\sigma^{3} & 0 \\
0 & -\sigma^{3}
\end{array}\right), \quad \Gamma^{t}=\left(\begin{array}{cc}
i \sigma^{1} & 0 \\
0 & i \sigma^{1}
\end{array}\right), \quad \Gamma^{x}=\left(\begin{array}{cc}
-\sigma^{2} & 0 \\
0 & \sigma^{2}
\end{array}\right), \quad \ldots
$$

The Dirac equation takes the form

$$
\left[\left(\partial_{r}+m \sqrt{g_{r r}} \sigma^{3}\right)-\sqrt{\frac{g_{r r}}{g_{t t}}}\left(\omega+q A_{t}\right) i \sigma^{2}-\left((-1)^{I} k \sqrt{\frac{g_{r r}}{g_{x x}}}-p \sqrt{g^{t t}} \partial_{r} A_{t}\right) \sigma^{1}\right] F_{I}=0
$$

with $I=1,2$. Decomposing $F_{I}$ into $F_{I}=\left(\mathcal{A}_{I}, \mathcal{B}_{I}\right)^{T}$, we can get the equation of motion for all the component as

$$
\begin{aligned}
& \left(\partial_{r}+m \sqrt{g_{r r}}\right) \mathcal{A}_{I}-\sqrt{\frac{g_{r r}}{g_{t t}}}\left(\omega+q A_{t}\right) \mathcal{B}_{I}-\left((-1)^{I} k \sqrt{\frac{g_{r r}}{g_{x x}}}-p \sqrt{g^{t t}} \partial_{r} A_{t}\right) \mathcal{B}_{I}=0 \\
& \left(\partial_{r}-m \sqrt{g_{r r}}\right) \mathcal{B}_{I}+\sqrt{\frac{g_{r r}}{g_{t t}}}\left(\omega+q A_{t}\right) \mathcal{A}_{I}-\left((-1)^{I} k \sqrt{\frac{g_{r r}}{g_{x x}}}-p \sqrt{g^{t t}} \partial_{r} A_{t}\right) \mathcal{A}_{I}=0 .
\end{aligned}
$$

Then defining $\xi_{I} \equiv \frac{\mathcal{A}_{I}}{\mathcal{B}_{I}}$, we can obtain the flow equation

$$
\left(\partial_{r}+2 m \sqrt{g_{r r}}\right) \xi_{I}-\left[v_{-}+(-1)^{I} k \sqrt{\frac{g_{r r}}{g_{x x}}}\right]-\left[v_{+}-(-1)^{I} k \sqrt{\frac{g_{r r}}{g_{x x}}}\right] \xi_{I}^{2}=0,
$$

where we have defined $v_{ \pm}=\sqrt{\frac{g_{r r}}{g_{t t}}}\left(\omega+q A_{t}\right) \pm p \sqrt{g^{t t}} \partial_{r} A_{t}$. For the convenience of numerical calculation later, we make a transformation $r=1 / u$, so that the flow equation (3.7) can be rewritten as

$$
\left(\sqrt{f} \partial_{u}-2 m u^{\frac{\theta}{2}-1}\right) \xi_{I}+\left[\frac{\tilde{v_{-}}}{u}+(-1)^{I} k\right]+\left[\frac{\tilde{v_{+}}}{u}-(-1)^{I} k\right] \xi_{I}^{2}=0
$$

with

$$
\tilde{v_{ \pm}}=\frac{u^{z}}{\sqrt{f}}\left(\omega+q A_{t}\right) \mp p u^{z-\theta / 2+1} \partial_{u} A_{t} .
$$

\subsection{Green's function}

\subsubsection{UV limit}

We first consider the UV limit of the Dirac equation. Equation (1.1) with hyperscaling violation gives $g_{r r}=r^{-\theta-2}, g_{t t}=r^{2 z-\theta}$ and $g_{x x}=g_{y y}=r^{2-\theta}$ in the UV limit. Therefore, the Dirac equation (3.4) becomes

$$
\left[\partial_{r}+m r^{-\frac{\theta}{2}-1} \sigma^{3}-r^{-1-z}(\omega+q \mu) i \sigma^{2}-\left((-1)^{I} k r^{-2}-p \mu(z-\theta) r^{\frac{3}{2} \theta-2 z-1}\right) \sigma^{1}\right] F_{I}=0 .
$$

Considering the allowed range of values of $\theta$ and $z$ in equation (2.12), we can reduce equation (3.10) in the limit of $r \rightarrow \infty$ to

$$
\left(\partial_{r}+\frac{m}{r^{\frac{\theta}{2}+1}} \sigma^{3}\right) F_{I} \approx 0 .
$$


For $\theta=0$, equation (3.11) give the following solution

$$
F_{I} \stackrel{r \rightarrow \infty}{\approx} b_{I} r^{m}\left(\begin{array}{l}
0 \\
1
\end{array}\right)+a_{I} r^{-m}\left(\begin{array}{l}
1 \\
0
\end{array}\right),
$$

which agrees well with the results in AdS or Lifshitz-AdS geometry. While for $\theta \neq 0$, the asymptotical behavior of $F_{I}$ becomes subtle. Firstly, equation (3.11) for $\theta \neq 0$ satisfies the solutions

$$
\mathcal{A}_{I}=a_{I} e^{\frac{2 m}{\theta} r^{-\frac{\theta}{2}}} \simeq a_{I}\left(1+\frac{2 m}{\theta} r^{-\frac{\theta}{2}}+\ldots\right), \quad \mathcal{B}_{I}=b_{I} e^{-\frac{2 m}{\theta} r^{-\frac{\theta}{2}}} \simeq b_{I}\left(1-\frac{2 m}{\theta} r^{-\frac{\theta}{2}}+\ldots\right) .
$$

Due to $\frac{2 m}{\theta} r^{-\frac{\theta}{2}} \rightarrow 0$ for $\theta>0$ in the limit of $r \rightarrow \infty$, we can deduce that at the leading order, the behavior of $F_{I}$ is

$$
F_{I} \stackrel{r \rightarrow \infty}{\approx} b_{I}\left(\begin{array}{l}
0 \\
1
\end{array}\right)+a_{I}\left(\begin{array}{l}
1 \\
0
\end{array}\right)
$$

which is the same asymptotical behavior as the case of zero mass in AdS (Lifshitz-AdS) geometry. According to the discussion in [7], it applies that we can choose either $a_{I}$ or $b_{I}$ as the source when we quantize Fermi field with different boundary conditions. In this work, we will choose $b_{I}$ as the source and $a_{I}$ as the response. Thus, in the regime of linear response, the boundary Green's functions can be extracted by $G_{I}=\frac{a_{I}}{b_{I}}$. If we define

$$
\xi_{I} \equiv \frac{\mathcal{A}_{I}}{\mathcal{B}_{I}}
$$

So the boundary Green's functions can be expressed in terms of $\xi_{I}$

$$
G(\omega, k)=\left(\begin{array}{cc}
G_{1} & 0 \\
0 & G_{2}
\end{array}\right)= \begin{cases}\lim _{r \rightarrow \infty} r^{2 m}\left(\begin{array}{cc}
\xi_{1} & 0 \\
0 & \xi_{2}
\end{array}\right), & \theta=0, \\
\lim _{r \rightarrow \infty}\left(\begin{array}{cc}
\xi_{1} & 0 \\
0 & \xi_{2}
\end{array}\right), & \theta>0 .\end{cases}
$$

Also, from equation (3.8), we can see that the Green function has the following symmetry

$$
G_{1}(\omega, k ; m, p)=G_{2}(\omega,-k ; m, p)
$$

\subsubsection{IR limit}

We then turn to the IR limit of the equations of motion. Since the near horizon geometry is $A d S_{2} \times \mathbb{R}^{2}$, we will use the metric (2.19). In the low energy frequency limit $\omega \rightarrow 0$, the Dirac equation takes the form

$$
\varsigma \partial_{\varsigma} F_{I}-\left[m L_{2} \sigma^{3}+\left((z-\theta) p \mu-(-1)^{I} k\right) L_{2} \sigma^{1}-i \sigma^{2} q(z-\theta) \mu L_{2}^{2}\right] F_{I}=0 .
$$

Note that we also choose the same Gamma matrices (3.3) but change $\Gamma^{\varsigma}=-\Gamma^{r}$ to reflect the orientation between the coordinates $r$ and $\varsigma$. As it was discussed in [7], equation (3.18) coincides with the equation of motion for spinor fields in $A d S_{2}$ background with masses

$$
\left[m, \tilde{m}_{I}=(z-\theta) p \mu-(-1)^{I} k\right]
$$



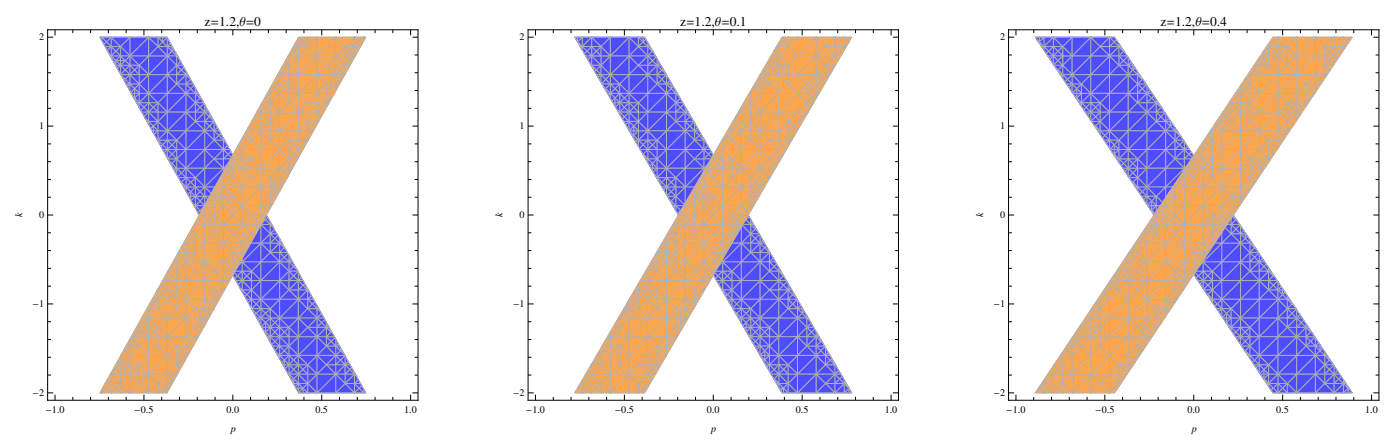

Figure 1. The oscillatory regions for $z=1.2 ; \theta=0$ (left), $z=1.2 ; \theta=0.1$ (middle) and $z=1.2$; $\theta=0.4$ (right). The orange region denotes the oscillatory region $\mathfrak{I}_{2}$ for $G_{22}(\omega, k)$ while the blue region corresponds to oscillatory region $\mathfrak{I}_{1}$ for $G_{11}(\omega, k)$.

where $\tilde{m}_{I}(I=1,2)$ are time-reversal violating mass terms. Then $F_{I}^{(0)}(\varsigma)$ is dual to the spinor operators $\mathbb{O}_{I}$ in the IR $C F T_{1}$ and the conformal dimensions of the operators are $\delta_{I}=\nu_{I}(k)+\frac{1}{2}$ with

$$
\nu_{I}(k)=\sqrt{\left(m^{2}+\tilde{m}_{I}^{2}\right) L_{2}^{2}-\left[(z-\theta) q \mu L_{2}^{2}\right]^{2}} \quad(I=1,2) .
$$

In (3.20) the Lifshitz and hyperscaling violation exponents as well as the coupling parameters like the dipole moment appear explicitly and they imprint their scalings in the IR limit.

There exists a range of momentum

$$
k \in \mathfrak{I}_{I}=\left[(-1)^{I}(z-\theta) p \mu-(z-\theta) q \mu L_{2},(-1)^{I}(z-\theta) p \mu+(z-\theta) q \mu L_{2}\right]
$$

in which $\nu(k)$ becomes pure imaginary. This region of momentum space is considered as the oscillatory region. If the Fermi momentum falls in this region, the peak will lose its meaning as a Fermi surface [7]. From the expression (3.21), it is obvious that for fixed $z$ and $\theta$, the oscillatory regions for the two-dimensional dual operator are coincident with $\mathfrak{I}_{1}=\mathfrak{I}_{2}=\left[-(z-\theta) q \mu L_{2},(z-\theta) q \mu L_{2}\right]$ for minimal coupling $p=0$, but they will separate when we turn on the dipole coupling. And when the dipole coupling satisfies $|p|>q L_{2}$, they will have no intersection. The separation behavior of the regimes $\mathfrak{I}_{1}$ and $\mathfrak{I}_{2}$ versus $p$ for various exponents can be seen in figure 1. It is obvious that the symmetry eq. (3.17) is well embodied in the figure, i.e., $\mathfrak{I}_{1}=-\mathfrak{I}_{2}$ for fixed dipole coupling. Another property we can see from eq. (3.21) and the figure 1 is that $\mathfrak{I}_{1}$ at $p$ coincides with $\mathfrak{I}_{2}$ at $-p$. The figure also shows that for larger hyperscaling exponent, the boundary of the oscillatory region is more smooth. We will see later that this behaviour will reflect a phase transition which occurs at a critical dipole coupling with the variation of the exponents.

Also following the matching method proposed in [7], we can match the inner and outer solutions in the matching region $(\varsigma \rightarrow 0$ and $\omega / \varsigma \rightarrow 0)$. We express the coefficients $a_{I}$ and $b_{I}$ in $(3.14)$ as

$$
\begin{aligned}
a_{I} & =\left[a_{I}^{(0)}+\omega a_{I}^{(1)}+\cdots\right]+\left[\tilde{a}_{I}^{(0)}+\omega \tilde{a}_{I}^{(1)}+\cdots\right] \mathcal{G}_{I}(k, \omega), \\
b_{I} & =\left[b_{I}^{(0)}+\omega b_{I}^{(1)}+\cdots\right]+\left[\tilde{b}_{I}^{(0)}+\omega \tilde{b}_{I}^{(1)}+\cdots\right] \mathcal{G}_{I}(k, \omega),
\end{aligned}
$$


where coefficients $a_{I}^{(n)}, \tilde{a}_{I}^{(n)}, b_{I}^{(n)}$ and $\tilde{b}_{I}^{(n)}$ are to be determined and

$$
\begin{aligned}
& \mathcal{G}_{I}(k, \omega)= \\
& \left\{e^{-i \pi \nu_{I}(k)} \frac{\Gamma\left(-2 \nu_{I}(k)\right) \Gamma\left(1+\nu_{I}(k)-i(z-\theta) q \mu L_{2}^{2}\right)\left[\left(m+i \tilde{m}_{I}\right) L_{2}-i(z-\theta) q \mu L_{2}^{2}-\nu_{I}(k)\right]}{\Gamma\left(2 \nu_{I}(k)\right) \Gamma\left(1-\nu_{I}(k)-i(z-\theta) q \mu L_{2}^{2}\right)\left[\left(m+i \tilde{m}_{I}\right) L_{2}-i(z-\theta) q \mu L_{2}^{2}+\nu_{I}(k)\right]}\right\} \omega^{2 \nu_{I}(k)}
\end{aligned}
$$

is the retarded Green functions of the dual operators $\mathbb{O}_{I}$. We see that the hyperscaling violation exponent and dipole coupling explicitly modify the boundary Green's function. More discussions on the above Green's function can be seen in [7] where it was found that (3.23) is only valid when $2 \nu_{I}(k)$ is not an integer. In the case when it is an integer, terms like $\omega^{n} \log (\omega)$ should be added.

Since the IR geometry of the charged geometry with hyperscaling violation is also $A d S_{2} \times \mathbb{R}^{2}$ as that in RN-AdS black brane and Lifshitz AdS black brane, we can easily derive the boundary conditions of $\xi$ at the horizon $r_{\mathrm{h}}=1$ for $\omega \neq 0$ and $\omega=0$ as

$$
\begin{cases}\xi_{I} \stackrel{r \rightarrow 1}{=} i & \text { for } \omega \neq 0, \\ \xi_{I} \stackrel{r \rightarrow 1}{=} \frac{m L_{2}-\nu_{I}(k)}{(z-\theta) q \mu L_{2}^{2}+\tilde{m}_{I} L_{2}} & \text { for } \omega=0 .\end{cases}
$$

\section{The effect of dipole coupling on the spectral function in the Fermionic system}

We numerically solve the flow equation (3.8) and read off the asymptotic values to extract the retarded Green functions. By studying the spectral function, we will explore the generation of a gap phase due to large enough dipole coupling in the bulk with different hyperscaling violation strength. Then, we also go to small dipole coupling to find out the Fermi momentum and the type of excitations near the Fermi surface. Our study will focus on taking $m=0$ and $q=0.5$.

\subsection{The emergence of the Mott gap}

We will calculate the density of states $A(\omega)$ by doing the integration of the Fermi spectral function $A(\omega ; k)=\operatorname{Tr}[\operatorname{Im} \mathrm{G}(\omega ; \mathrm{k})]$ over $k$. The effects of the dynamical Lifshitz exponent on the holographic fermionic systems and the emergence of the Mott gap were discussed in [39-41]. It was found that the gap opens easier for the larger Lifshitz exponent. Here we will mainly focus on the effects of the hyperscaling exponent.

For comparison, we first show the results of the gap emergence due to the dipole coupling in a RN-AdS black hole background, i.e., with $z=1$ and $\theta=0$. In figure 2, we show the results of $\operatorname{Im} G_{22}$ for $p=0$ and $p=6$. The left plot is for $p=0$, where the quasi-particle-like peak at $\omega=0$ indicates a Fermi surface near which the low energy excitation is non-Fermi liquid type [5]. The right plot is for taking $p=6$ where an explicit gap is around $\omega=0$. This is the dipole coupling effect first studied in $[8,9]$.

Then we turn on hyperscaling violation $\theta$ to consider its effect on the dual field theory. The Green functions with fixed $z=1.2$ for different $\theta$ are shown in figure 3. Comparing 

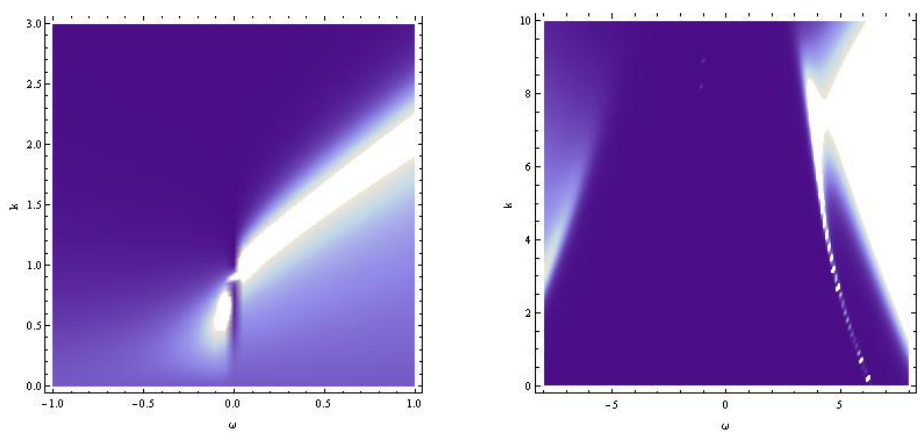

Figure 2. $\operatorname{Im}\left[G_{22}(\omega, k)\right]$ for $p=0$ (left plane) and $p=6$ (right plane) in RN-AdS background.
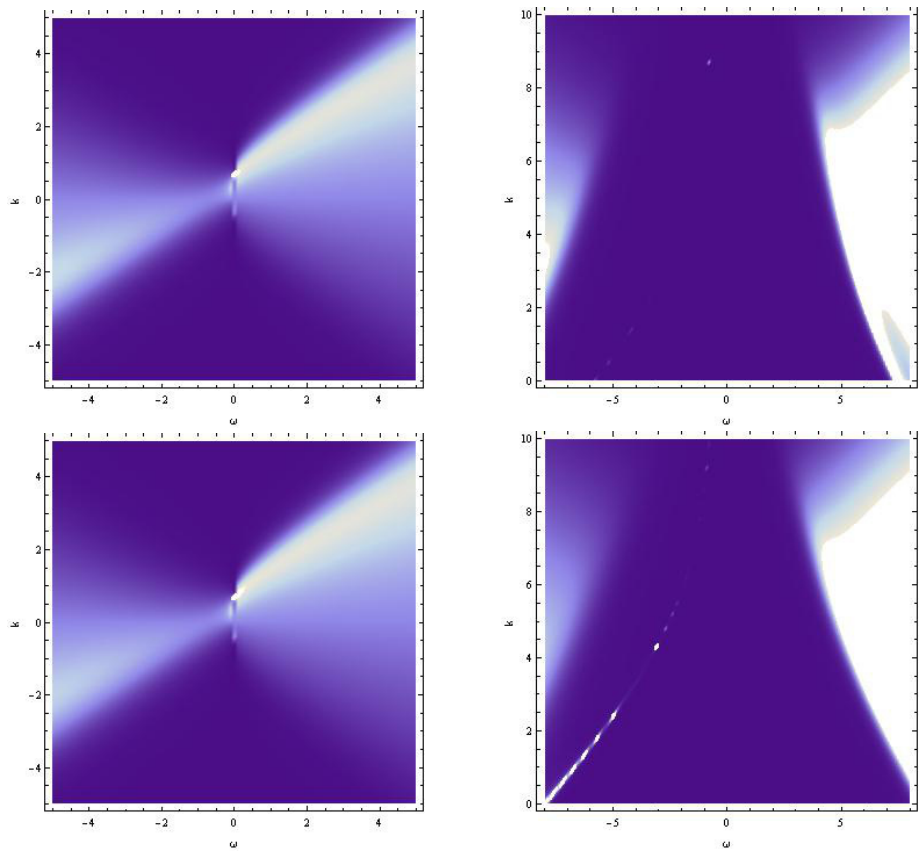

Figure 3. The density plots of $\operatorname{Im}\left[G_{22}(\omega, k)\right]$ for $p=0$ (left plane) and $p=6$ (right plane). The exponents for the up plane are $z=1.2$ and $\theta=0.1$ while the bottom plane are for $z=1.2$ and $\theta=0.4$.

the plots in figure 3, we find that for a fixed value of the dipole coupling, the larger hyperscaling violation exponent introduces smaller gap, which implies that in the theory with hyperscaling violation the Mott gap phase is hard to be formed. This feature is explicitly shown in figure 4, where we present the density of states near the chemical potential with $p$ by changing the hyperscaling violation exponent. Each plot shows that as the dipole coupling is strengthened, the spectral function will be suppressed near the zero frequency, then a gap will open at some critical value, accompanying spectral weight transfer from positive frequency band to negative band.

It is important to note that in figure 3 an explicit band is shown to be generated which disperses as $k$ increases. This band is very important because it contributes to the spectral function, especially the spectral weight transfer at negative enough frequency. The mechanism of the generation of this band is still called for. 

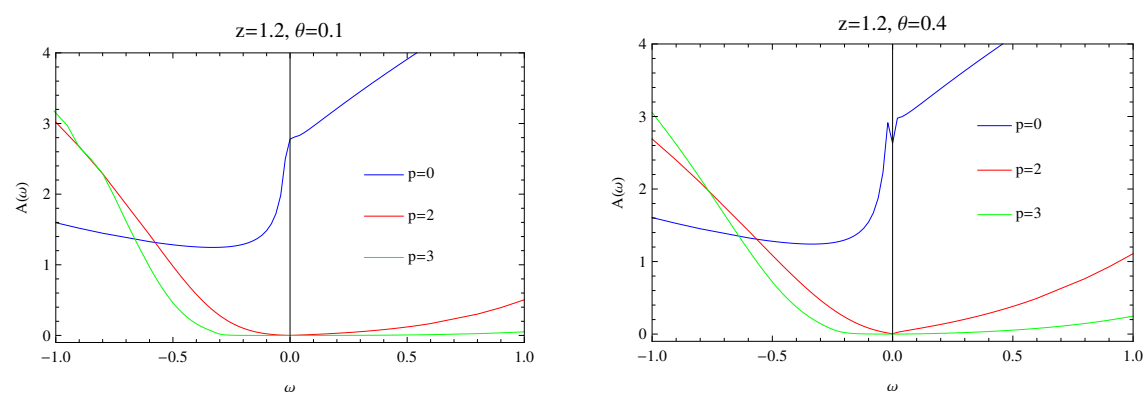

Figure 4. The behaviour of spectral function $A(\omega)$ with $\omega$ for $z=1.2$. We set $\theta=0.1$ (left plane) and $\theta=0.4$ (right plane) respectively.

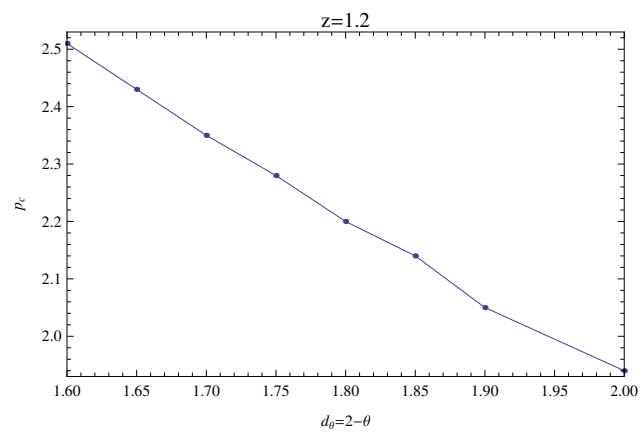

Figure 5. The dependence of $p_{\mathrm{c}}$ for the gap opening on the effective dimension $d_{\theta}=d-\theta$ with $z=1.2$. Here in our case we have $d=2$.

Carrying out some exact calculations to define the gap when the spectral function is below $\sim 0.0001$, we determine that the gap opens at $p_{\mathrm{c}} \simeq 1.94$ for $z=1.2$ and $\theta=0$, $p_{\mathrm{c}} \simeq 2.05$ for $z=1.2$ and $\theta=0.1$, and $p_{\mathrm{c}} \simeq 2.51$ for $z=1.2$ and $\theta=0.4$. These results show that as $\theta$ increases, $p_{\mathrm{c}}$ becomes larger, which means that the gap is more difficult to be generated dynamically.

This is a very interesting result. While the anisotropic Lifshitz scaling tends to decrease the critical value of the dipole moment $p_{\mathrm{c}}$, the increase of Lifshitz exponent can make the Mott gap open up more easily [39-41]. However, the increase of the hyperscaling violation factor plays the opposite role, which makes it harder for the Mott insulating phase to appear. This behaviour can be understood as follows. As we discussed in the introduction, the hyperscaling violation factor introduces an effective dimension $d_{\theta}=d-\theta$ into the theory with $d=2$ in our holographic model. So larger hyperscaling violation factor corresponds to lower effective dimension. Then if one looks at the flow equation, the critical $p$ is larger for lower dimension, because the spacetime dimension compensates the effect of $p$. This behaviour was observed for the first time in [13]. Furthermore, we get the dependence of $p_{\mathrm{c}}$ for the gap opening on the effective dimension $d_{\theta}$ with $z=1.2$ in figure 5 . It seems that in systems with lower effective dimension the Mott gap phase is harder to emerge.

\subsection{The formation of the Fermi surface and the type of low energy excitations}

In this subsection, we will turn to discuss the case with $p<p_{\mathrm{c}}$. We intend to see the effect of the dipole coupling on the Fermi surface as well as its type, and investigate how 

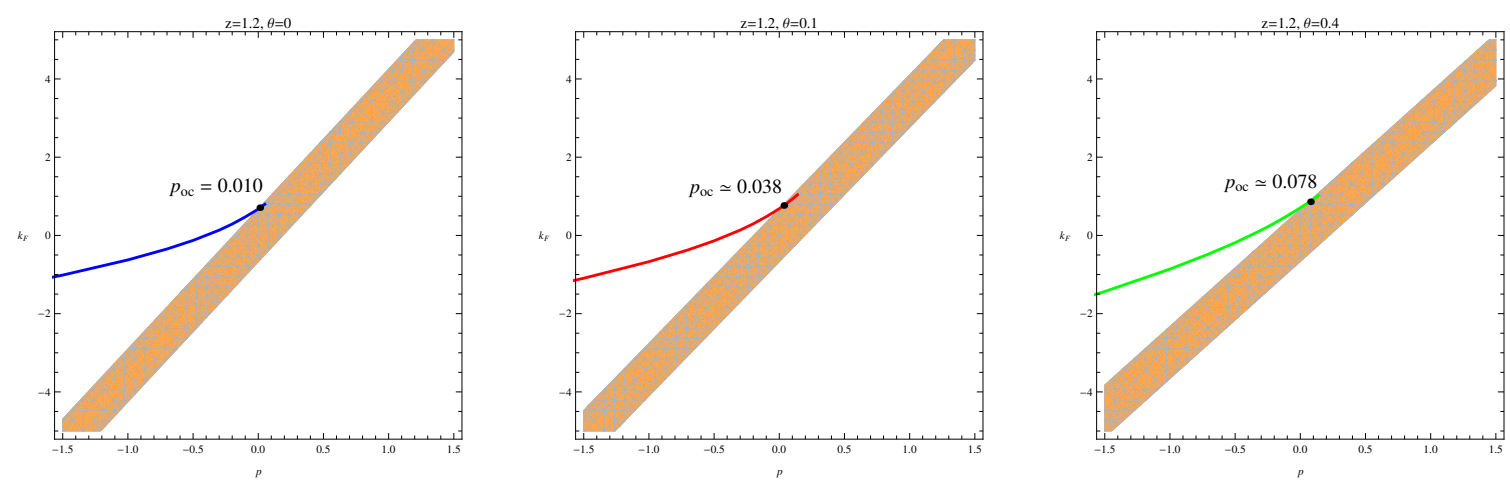

Figure 6. The momentum of Fermi surface corresponding to $G_{2}$ for small dipole coupling. The orange band denote the oscillatory region of $G_{2}$. The parameters from left to right are $z=1.2$ and $\theta=0, z=1.2$ and $\theta=0.1, z=1.2$ and $\theta=0.4$ respectively.

\begin{tabular}{|c|c|c|c|c|c|c|c|}
\hline$p$ & -1.5 & -1 & -0.5 & 0 & 0.02 & 0.05 & 0.1 \\
\hline$z=1.2, \theta=0$ & -1.0141 & -0.6262 & -0.1279 & 0.6812 & - & - & - \\
\hline$z=1.2, \theta=0.1$ & -1.0942 & -0.6713 & -0.1383 & 0.6883 & 0.7274 & - & - \\
\hline$z=1.2, \theta=0.4$ & -1.4334 & -0.8601 & -0.1853 & 0.7084 & 0.7490 & 0.8120 & - \\
\hline
\end{tabular}

Table 1. The Fermi momentum with different $p$ for the various parameters. Here "—" denote the Fermi momentum can not represent the Fermi surface.

the Fermi surface enters into the oscillating region, losing its meaning in the background with hyperscaling violation. To study the solutions explicitly, we will numerically solve the Dirac equations to determine where the system possesses a Fermi surface and the type of excitations.

Figure 6 displays our findings for the momentum of Fermi surface as well as its behaviour entering into the oscillatory regions with various exponents. In the figure we can see that as the hyperscaling violation exponent increases, the critical dipole coupling $p_{o c}$ which makes the Fermi momentum to enter the oscillatory region becomes larger due to the smoother oscillating boundary. This behavior is expected the same as that of $p_{\mathrm{c}}$ to open the Mott gap. The values of the critical dipole couplings are $p_{o c} \simeq 0.010$ for $z=1.2$ and $\theta=0, p_{o c} \simeq 0.038$ for $z=1.2$ and $\theta=0.1$ while $p_{o c} \simeq 0.078$ for $z=1.2$ and $\theta \simeq 0.4$. In all cases, when $p>p_{o c}$, the Fermi momentum will lose its meaning of Fermi surface. When $p$ is smaller than $p_{o c}$, the Fermi momentum increases as the dipole coupling becomes large and then enters into the oscillatory region, which is consistent with the observation in [9]. In addition, we show the samples of Fermi momentum with different $p$ for various parameters in table 1. For $p$ deviates away from $p_{o c}$, we see that the Fermi momentum is smaller for larger hyperscaling exponents. While near the oscillating boundary and for the minimal coupling, the Fermi momentum is larger for bigger exponents due to the lower effective dimension as we discussed in [38]. This behavior near the oscillatory region can be attributed to the smooth boundary of the region with larger hyperscaling exponent.

Having the Fermi momentum, we can calculate the dimensionless scaling $\nu_{2}(k)$ in terms of the expression (3.20). As discussed in [5], imaginary $\nu_{I}\left(k=k_{F}\right)$ corresponds to 


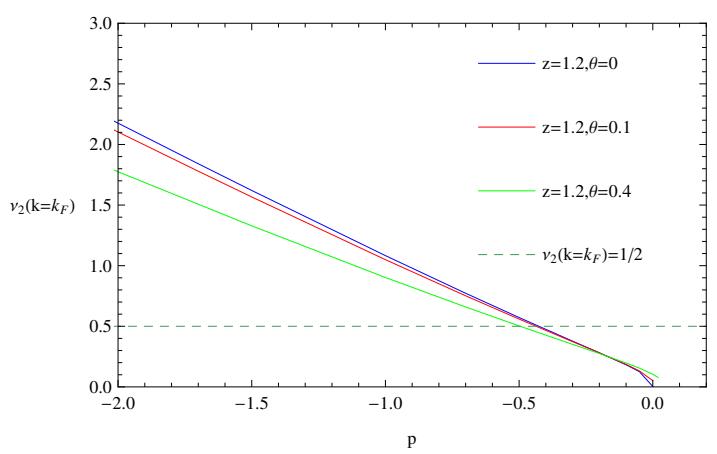

Figure 7. $\nu_{2}(k)$ changing with the dipole coupling for various parameter. We mark the marginal Fermi type with dashed line.

"log oscillatory" solutions as we emphasized before. When $\nu_{I}\left(k=k_{F}\right)<1 / 2$, the pole of $G_{R}$ corresponds to an unstable quasi-particle which is identified as a non-Fermi fluid. With the value $\nu_{I}\left(k=k_{F}\right)=1 / 2$, the excitations are of marginal Fermi fluid type. For $\nu_{I}\left(k=k_{F}\right)>1 / 2$ the dispersion relation is linear denoting the Fermi fluid.

The results of $\nu_{2}(k)$ changing with the dipole coupling are shown in figure 7 . With all choices of exponents, there is a phase transition from Fermi liquid to marginal Fermi liquid then to non-Fermi liquid as the dipole coupling becomes stronger. Combining figure 7 and figure 6, we can cconclude that the system can not show a Fermi surface unless $p<$ 0.010, thereafter the types of excitations near the Fermi surface are non-Fermi liquid for $-0.425<p<0.010$, marginal Fermi liquid for $p \simeq-0.425$ and Fermi liquid for $p<-0.425$ when $z=1.2$ and $\theta=0$. Taking the same $z$, for example $z=1.2$, we find that different hyperscaling values $\theta=0.1$ and $\theta=0.4$ lead to the values of marginal Fermi liquid $p \simeq-0.441$ and $p \simeq-0.496$, respectively. This tells us that smaller dipole coupling corresponds to Fermi Liquid while larger one corresponds to non-Fermi liquid ending by the critical value $p_{o c}$.

With $z=1.2$, for larger hyperscaling exponent, the phase transition happens at more negative dipole coupling. We can understand the phenomenon as follow. As we mentioned earlier that larger hyperscaling violation recedes the effective dimension $d_{\theta}=2-\theta$, which always appears in the form $p\left(z+d_{\theta}-2\right)$ in the flow equation and compensate the effect of $p$ [13]. So it is reasonable that lower effective dimension corresponds to more negative dipole coupling for the phase transition. Combining the phenomena that larger $\theta$ needs more negative $p$ for the phase transition, we observed that the generation of gap with larger $\theta$ calls for stronger positive $p_{\mathrm{c}}$ in the last subsection. It would be interesting to check the duality under the transformation $p \rightarrow-p$ proposed in [20] for the hyperscaling model which will be carried out in the next section.

\section{The zeros-poles duality of the holographic system}

In this section we will study the behaviour of $\operatorname{det} G_{R}$ in different dipole couplings with the aim to see if in holographic theories with hyperscaling violation the duality between zeros 
and poles under $p \rightarrow-p$ found in [20] still holds. Following [20] we define

$$
\zeta_{I}=\frac{1}{\xi_{I}}
$$

which satisfies the following equation

$$
\left(\sqrt{f} \partial_{u}+2 m u^{\frac{\theta}{2}-1}\right) \zeta_{I}-\left[\frac{\tilde{v_{-}}}{u}+(-1)^{I} k\right] \zeta_{I}^{2}-\left[\frac{\tilde{v_{+}}}{u}-(-1)^{I} k\right]=0 .
$$

Comparing the above equation with equation (3.8), we find that the equation of $\zeta_{I}$ coincides with the equation of $\xi_{I}$ under the transformation of $(m, k, p) \rightarrow(-m,-k,-p)$. Then considering the symmetry of the Green function (3.17), we can get the relation

$$
\operatorname{det} G_{R}(\omega=0, k ; m, p)=\operatorname{det} G_{R}(\omega=0,-k ; m, p)=\frac{1}{\operatorname{det} G_{R}(\omega=0, k ;-m,-p)} .
$$

The formula with $m=0$ coincides with the expression (30) in [20] and its value is one for $p=0[7]$.

Note that a pole of $G_{I}$ at $\omega=0$ is not necessarily a pole of the $\operatorname{determinant} \operatorname{det} G_{R}=$ $G_{1} G_{2}$. It is known [42] that in the conventional case, $p=0$, $\operatorname{det} G_{R}(\omega=0, k ; p=0)=-1$, therefore it possesses neither poles nor zeroes. This is because poles (zeroes) of $G_{1}$ are cancelled by zeroes (poles) of $G_{2}$ at the same momentum. It was showed in [20] that this coincidence of poles and zeroes is lifted when the dipole coupling is turned on, resulting in poles and zeroes of $\operatorname{det} G_{R}$.

We firstly turn off the hyperscaling violation factor. The result is showed in the left panel of figure 8 with $p=2.5$, which we reproduce the corresponding result of [20] with $p=5$. The behaviour of $\operatorname{det} G$ with non-zero hyperscaling violation is also presented in the middle and right panel of figure 8. For $\theta=0.1$, the zero for the real part of $\operatorname{det} G$ is around $k \simeq 1.78$ with $p=2.5$, corresponding to a pole at the same momentum with $p=-2.5$. While for $\theta=0.4$, the momentum related to the zero-pole duality is $k \simeq 2.42$. We see that the hyperscaling violation does not break the zero-pole duality under the transformation $p \rightarrow-p$. This is expected because the zeros-poles duality is a reflection of the symmetries of the Green's function and it should not be related to the dimensionality of these theories.

\section{Conclusions and discussion}

We have studied the behaviour of a holographic fermionic system with a charged black brane with hyperscaling violation in the bulk in the presence of dipole interaction between a massless fermion and a gauge field. We found that the holographic system possesses a robust phase diagram including Fermi and non-Fermi liquids, marginal Fermi liquid, log oscillatory phase and an insulating Mott phase. These various phases are controlled by the strength of the dipole coupling and the hyperscaling violation exponent which play the role of the order parameters in the holographic system $[8,9]$.

These phases can be identified by studying the behaviour of the Green's function. Considering the IR and the UV limits of the Green's function, we used the matching 

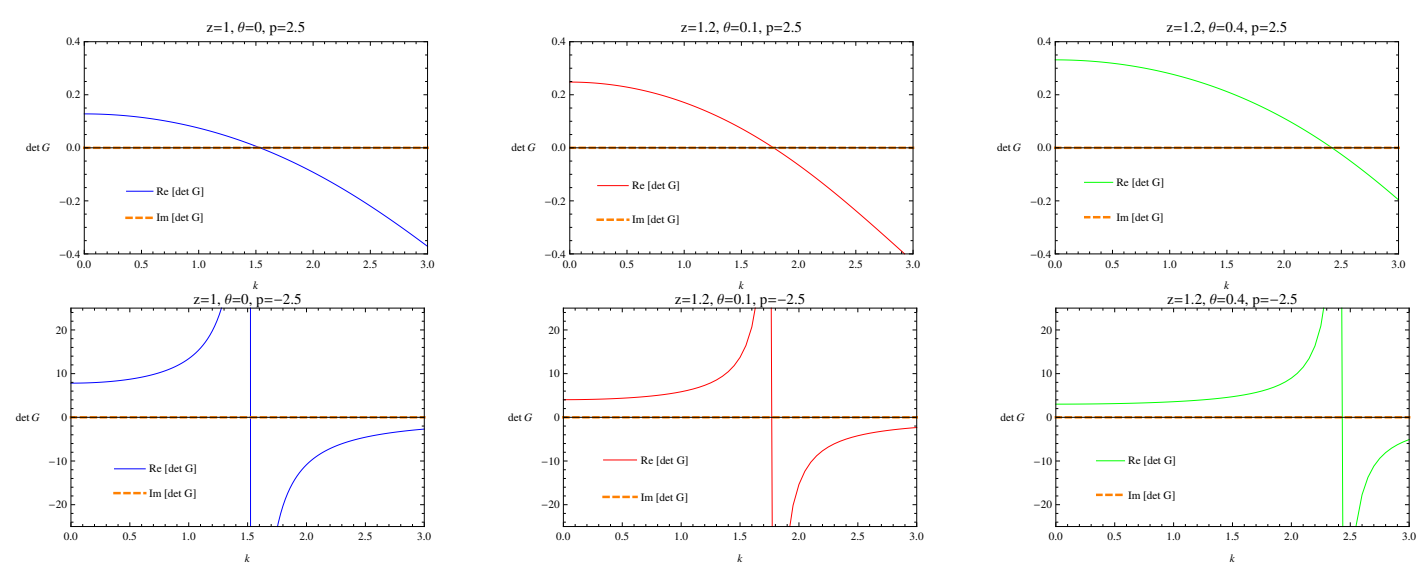

Figure 8. The real and imaginary parts of $\operatorname{det} G$ depending on the momentum for $p=2.5$ with hyperscaling violation.

method [43] in the near horizon geometry to obtain the analytical expressions of the UV Green's function and the dispersion relation.

We found that as the hyperscaling violation exponent is increased the critical value of the dipole moment $p_{\mathrm{c}}$ for a Mott gap to be generated is also increased, which makes it harder for the insulating phase to be formed. This is attributed to the fact that a larger hyperscaling violation exponent corresponds to a lower effective dimensionality of the system and this change compensates the increase in critical dipole coupling $p_{\mathrm{c}}$. Then, we investigated the existence of the Fermi surface and the disperse relation near the Fermi surface. We found that as $p$ increases, the liquid changes from Fermi type, marginal Fermi type to non-Fermi type, then the Fermi surface disappears in the Log-oscillatory region. The phase transition between the types of liquid happens at negative dipole coupling and the larger hyperscaling violation will result in more negative dipole coupling.

A pole is indicative of a (non-) Fermi fluid while a zero is responsible for an insulating phase. It is the coexistence of both that underlies the various phases of the liquid. We showed that the duality found in [20] relating systems of opposite dipole coupling strength $p$ also persists in holographic phase with hyperscaling violation exponent.

It would be interesting to consider a Lorentz violating boundary term into the bulk action instead of the standard boundary condition. It was discussed in [44] that with the Lorentz violating boundary term, the dual field theory has a holographic non-relativistic fixed point, possessing a flat band of gapless excitation. With the minimal coupling, we have also observed a flat band in the boundary theory dual to hyperscaling violation background in [38]. So it is important to see how the hyperscaling violation will influence the dipole effect in the dual non-relativistic fermionic system. This study is underway.

Another possible direction is to calculate the holographic entanglement entropy in our theory. The entanglement entropy $[45,46]$ was proved to be a powerful tool in counting the degrees of freedom available in a holographic system. In [47] it was found that the holographic entanglement entropy in the superconducting phase is less than that in the normal phase due to the fact that Cooper pairs had been formed so that fewer degrees of freedom 
are aleft(see also [48]). Near the contact interface of the superconductor to normal metal, the entanglement entropy could have higher value in the superconducting phase due to the proximity effect: the leakage of Cooper pairs to the normal phase results in more freedom in the superconductor side near the interface. It would be interesting to generalize previous study and discuss the holographic entanglement entropy in a holographic fermionic system with a dipole coupling and hyperscaling violation. This can give important information on the various phases of the system. To carry out such a study, we need a fully back-reacted solution of the Einsten-Maxwell-Dirac system. We will report results on this topic in the future.

\section{Acknowledgments}

X.M.K. is indebted to Li-Qing Fang for helping to run parts of our Mathematica programs in his computer. X.M.K. and E.P. are supported by ARISTEIA II action of the operational programme education and long life learning which is co-funded by the European Union (European Social Fund) and National Resources. X.M.K. is also supported by the Chilean FONDECYT Grants No. 3150006. B.W. and J.P.W. are supported by the Natural Science Foundation of China. J.P.W. is also supported by Program for Liaoning Excellent Talents in University (No. LJQ2014123).

Open Access. This article is distributed under the terms of the Creative Commons Attribution License (CC-BY 4.0), which permits any use, distribution and reproduction in any medium, provided the original author(s) and source are credited.

\section{References}

[1] J.M. Maldacena, The Large-N limit of superconformal field theories and supergravity, Int. J. Theor. Phys. 38 (1999) 1113 [Adv. Theor. Math. Phys. 2 (1998) 231] [hep-th/9711200] [INSPIRE].

[2] S.S. Gubser, I.R. Klebanov and A.M. Polyakov, A Semiclassical limit of the gauge/string correspondence, Nucl. Phys. B 636 (2002) 99 [hep-th/0204051] [InSPIRE].

[3] E. Witten, Anti-de Sitter space and holography, Adv. Theor. Math. Phys. 2 (1998) 253 [hep-th/9802150] [INSPIRE].

[4] S.-S. Lee, A Non-Fermi Liquid from a Charged Black Hole: A Critical Fermi Ball, Phys. Rev. D 79 (2009) 086006 [arXiv:0809.3402] [INSPIRE].

[5] H. Liu, J. McGreevy and D. Vegh, Non-Fermi liquids from holography, Phys. Rev. D 83 (2011) 065029 [arXiv:0903.2477] [INSPIRE].

[6] M. Cubrovic, J. Zaanen and K. Schalm, String Theory, Quantum Phase Transitions and the Emergent Fermi-Liquid, Science 325 (2009) 439 [arXiv:0904.1993] [INSPIRE].

[7] T. Faulkner, H. Liu, J. McGreevy and D. Vegh, Emergent quantum criticality, Fermi surfaces and $A d S_{2}$, Phys. Rev. D 83 (2011) 125002 [arXiv:0907.2694] [INSPIRE].

[8] M. Edalati, R.G. Leigh and P.W. Phillips, Dynamically Generated Mott Gap from Holography, Phys. Rev. Lett. 106 (2011) 091602 [arXiv: 1010.3238] [InSPIRE]. 
[9] M. Edalati, R.G. Leigh, K.W. Lo and P.W. Phillips, Dynamical Gap and Cuprate-like Physics from Holography, Phys. Rev. D 83 (2011) 046012 [arXiv:1012.3751] [INSPIRE].

[10] D. Guarrera and J. McGreevy, Holographic Fermi surfaces and bulk dipole couplings, arXiv:1102.3908 [INSPIRE].

[11] J.P. Wu, Holographic fermions in charged Gauss-Bonnet black hole, JHEP 07 (2011) 106 [arXiv: 1103.3982] [INSPIRE].

[12] X.-M. Kuang, B. Wang and J.-P. Wu, Dynamical gap from holography in the charged dilaton black hole, Class. Quant. Grav. 30 (2013) 145011 [arXiv:1210.5735] [INSPIRE].

[13] X.-M. Kuang, B. Wang and J.-P. Wu, Dipole Coupling Effect of Holographic Fermion in the Background of Charged Gauss-Bonnet AdS Black Hole, JHEP 07 (2012) 125 [arXiv:1205.6674] [INSPIRE].

[14] L.Q. Fang, X.-H. Ge and X.-M. Kuang, Holographic fermions with running chemical potential and dipole coupling, Nucl. Phys. B 877 (2013) 807 [arXiv: 1304.7431] [INSPIRE].

[15] J.-P. Wu, Some properties of the holographic fermions in an extremal charged dilatonic black hole, Phys. Rev. D 84 (2011) 064008 [arXiv:1108.6134] [INSPIRE].

[16] W.-J. Li and J.-P. Wu, Holographic fermions in charged dilaton black branes, Nucl. Phys. B 867 (2013) 810 [arXiv: 1203.0674] [INSPIRE].

[17] W.-J. Li, R. Meyer and H.-b. Zhang, Holographic non-relativistic fermionic fixed point by the charged dilatonic black hole, JHEP 01 (2012) 153 [arXiv:1111.3783] [INSPIRE].

[18] W.-J. Li and H.-b. Zhang, Holographic non-relativistic fermionic fixed point and bulk dipole coupling, JHEP 11 (2011) 018 [arXiv:1110.4559] [INSPIRE].

[19] Y. Ling, P. Liu, C. Niu, J.-P. Wu and Z.-Y. Xian, Holographic fermionic system with dipole coupling on Q-lattice, JHEP 12 (2014) 149 [arXiv:1410.7323] [INSPIRE].

[20] J. Alsup, E. Papantonopoulos, G. Siopsis and K. Yeter, Duality between zeroes and poles in holographic systems with massless fermions and a dipole coupling, Phys. Rev. D 90 (2014) 126013 [arXiv: 1404 .4010] [INSPIRE].

[21] G. Vanacore and P.W. Phillips, Minding the Gap in Holographic Models of Interacting Fermions, Phys. Rev. D 90 (2014) 044022 [arXiv:1405.1041] [INSPIRE].

[22] S. Kachru, X. Liu and M. Mulligan, Gravity duals of Lifshitz-like fixed points, Phys. Rev. D 78 (2008) 106005 [arXiv:0808.1725] [InSPIRE].

[23] B. Gouteraux and E. Kiritsis, Generalized Holographic Quantum Criticality at Finite Density, JHEP 12 (2011) 036 [arXiv:1107.2116] [InSPIRE].

[24] L. Huijse, S. Sachdev and B. Swingle, Hidden Fermi surfaces in compressible states of gauge-gravity duality, Phys. Rev. B 85 (2012) 035121 [arXiv:1112.0573] [INSPIRE].

[25] X. Dong, S. Harrison, S. Kachru, G. Torroba and H. Wang, Aspects of holography for theories with hyperscaling violation, JHEP 06 (2012) 041 [arXiv: 1201.1905] [INSPIRE].

[26] C. Charmousis, B. Gouteraux, B.S. Kim, E. Kiritsis and R. Meyer, Effective Holographic Theories for low-temperature condensed matter systems, JHEP 11 (2010) 151 [arXiv: 1005.4690] [INSPIRE].

[27] D.S. Fisher, Scaling and critical slowing down in random-field Ising systems, Phys. Rev. Lett. 56 (1986) 416 [INSPIRE].

[28] M. Taylor, Non-relativistic holography, arXiv:0812.0530 [INSPIRE].

[29] D.-W. Pang, A Note on Black Holes in Asymptotically Lifshitz Spacetime, Commun. Theor. Phys. 62 (2014) 265 [arXiv:0905.2678] [INSPIRE]. 
[30] J. Tarrio and S. Vandoren, Black holes and black branes in Lifshitz spacetimes, JHEP 09 (2011) 017 [arXiv: 1105.6335] [INSPIRE].

[31] N. Ogawa, T. Takayanagi and T. Ugajin, Holographic Fermi Surfaces and Entanglement Entropy, JHEP 01 (2012) 125 [arXiv:1111.1023] [INSPIRE].

[32] M.M. Wolf, Violation of the entropic area law for Fermions, Phys. Rev. Lett. 96 (2006) 010404 [quant-ph/0503219] [INSPIRE].

[33] B. Swingle, Entanglement Entropy and the Fermi Surface, Phys. Rev. Lett. 105 (2010) 050502 [arXiv: 0908.1724] [inSPIRE].

[34] U. Gürsoy, E. Plauschinn, H. Stoof and S. Vandoren, Holography and ARPES Sum-Rules, JHEP 05 (2012) 018 [arXiv: 1112.5074] [INSPIRE].

[35] M. Alishahiha, M.R. Mohammadi Mozaffar and A. Mollabashi, Fermions on Lifshitz Background, Phys. Rev. D 86 (2012) 026002 [arXiv:1201.1764] [InSPIRE].

[36] M. Alishahiha, E. O Colgain and H. Yavartanoo, Charged Black Branes with Hyperscaling Violating Factor, JHEP 11 (2012) 137 [arXiv:1209.3946] [INSPIRE].

[37] L.Q. Fang, X.-H. Ge and X.-M. Kuang, Holographic fermions in charged Lifshitz theory, Phys. Rev. D 86 (2012) 105037 [arXiv:1201.3832] [INSPIRE].

[38] X.-M. Kuang, E. Papantonopoulos, B. Wang and J.-P. Wu, Formation of Fermi surfaces and the appearance of liquid phases in holographic theories with hyperscaling violation, JHEP 11 (2014) 086 [arXiv: 1409.2945] [INSPIRE].

[39] J.-P. Wu, Holographic fermions on a charged Lifshitz background from Einstein-Dilaton-Maxwell model, JHEP 03 (2013) 083 [INSPIRE].

[40] J.-P. Wu, Emergence of gap from holographic fermions on charged Lifshitz background, JHEP 04 (2013) 073 [INSPIRE].

[41] J.-P. Wu, The charged Lifshitz black brane geometry and the bulk dipole coupling, Phys. Lett. B 728 (2014) 450 [INSPIRE].

[42] T. Faulkner, N. Iqbal, H. Liu, J. McGreevy and D. Vegh, From Black Holes to Strange Metals, arXiv: 1003.1728 [INSPIRE].

[43] N. Iqbal and H. Liu, Real-time response in AdS/CFT with application to spinors, Fortsch. Phys. 57 (2009) 367 [arXiv:0903.2596] [INSPIRE].

[44] J.N. Laia and D. Tong, A Holographic Flat Band, JHEP 11 (2011) 125 [arXiv:1108.1381] [INSPIRE].

[45] S. Ryu and T. Takayanagi, Holographic derivation of entanglement entropy from AdS/CFT, Phys. Rev. Lett. 96 (2006) 181602 [hep-th/0603001] [INSPIRE].

[46] S. Ryu and T. Takayanagi, Aspects of Holographic Entanglement Entropy, JHEP 08 (2006) 045 [hep-th/0605073] [inSPIRE].

[47] X.-M. Kuang, E. Papantonopoulos and B. Wang, Entanglement Entropy as a Probe of the Proximity Effect in Holographic Superconductors, JHEP 05 (2014) 130 [arXiv:1401.5720] [INSPIRE].

[48] T. Albash and C.V. Johnson, Holographic Studies of Entanglement Entropy in Superconductors, JHEP 05 (2012) 079 [arXiv:1202.2605] [INSPIRE].

[49] S.A. Hartnoll, J. Polchinski, E. Silverstein and D. Tong, Towards strange metallic holography, JHEP 04 (2010) 120 [arXiv:0912.1061] [INSPIRE]. 